\title{
Protective effects of glucosamine-kynurenic acid after compression-induced spinal cord injury in the rat
}

\author{
Research Article
}

Andrea Korimová ${ }^{1}$, Dáša Cížková ${ }^{1}$, Jozsef Toldi ${ }^{2}$ László Vécsei ${ }^{3}$, Ivo Vanický1,*

1'Institute of Neurobiology, Slovak Academy of Sciences, 04001 Košice, Slovak Republic

2Department of Physiology, Anatomy and Neuroscience, University of Szeged, H-6726 Szeged, Hungary

${ }^{3}$ Department of Neurology, Albert Szent-Györgyi Clinical Center, University of Szeged, H-6725 Szeged, Hungary

Received 25 May 2012; Accepted 30 August 2012

Abstract: Kynurenic acid (KYNA), a metabolite of the essential amino acid L-tryptophan, is a broad spectrum antagonist of excitatory amino acid receptors, which have also anticonvulsant and neuroprotective properties. After spinal cord injury (SCl), excitotoxicity is considered to play a significant role in the processes of secondary tissue destruction in both grey and white matter of the spinal cord. In this study, we have tested the potential therapeutic effect of glucosamine-kynurenic acid, administered after experimental compressioninduced SCl in the rat. Spinal application of glucosamine-kynurenic acid continually for $24 \mathrm{hr}$ after experimental SCl resulted in improved motor function recovery, beginning from the first week of evaluation and continuing until the end of the study (4 weeks). After 4 weeks' survival, quantitative morphometric analysis of the spinal cord showed that glucosamine-kynurenic acid treatment was associated with improved tissue preservation at the lesion site. These findings indicate that spinal application of glucosaminekynurenic acid is neuroprotective and improves the outcome even when administered after spinal trauma. Our results suggest that the treatments initiated in early posttraumatic period can alleviate secondary injury and improve the final outcome after SCI.

Keywords: Spinal cord injury • Excitotoxicity $\bullet$ NMDA receptor • Kynurenic acid

(C) Versita Sp. z 0.0 .

\section{Introduction}

Traumatic spinal cord injury (SCI) is usually caused by excessive forces taking effect on vertebral column. Spinal cord tissues are damaged by displaced bone fragments, intervertebral discs or ligaments. After contusive injury to the spinal cord, pathological changes develop as a two-stage process $[1,2]$. The primary injury is caused immediately by mechanical damage to neural tissue (including blood vessels), and is followed by secondary processes, leading to posttraumatic autodestruction of spinal cord tissue [3-5]. The mechanisms of secondary injury are not completely understood, but include ischemia, biochemical changes and inflammatory processes contributing to gradual extension of the primary lesion. Histological observations indicate that secondary destruction takes place within the course of days and even weeks after injury [1,5], and that the enlargement of the lesion progresses from the center to the periphery of the spinal cord [6]. Progressive secondary injury involves gradual destruction of the white matter that can play an important role in the final functional deficit after $\mathrm{SCl}$ [7]. The detailed understanding of the molecular processes involved in secondary injury is therefore of great importance in $\mathrm{SCl}$ research. It would enable more specific therapeutic interventions aimed at minimizing the tissue loss at the injury site.

Studies on the dynamics of the development of secondary changes indicate that the major enlargement of the primary lesion occurs within the first 24 hours after injury. We have therefore proposed that treatments 
aimed at diminishing secondary tissue injury should be applied during the first 24 hours after trauma [6,8].

In animal experiments, several drug treatment studies reported promising results [9]. However, almost none of them have been found effective in clinical settings. So far, only methylprednisolone (MP), a potent synthetic glucocorticoid, has been shown effective at reducing neurological impairment after acute spinal cord trauma in clinical studies [10]. However, controversies still exist about its real therapeutic effect. Systemic administration of high doses of MP is associated with serious side-effects, and its standard use after $\mathrm{SCl}$ remains a matter of debate [11].

Excitatory amino acids (EAA) play an important role in the pathomechanisms of secondary injury after SCl. After trauma, local concentrations of several amino acids are significantly increased [12]. In brain ischemia and trauma studies, EAA antagonists have been the most potent neuroprotectants [13,14]. Kynurenic acid (KYNA) is an endogeneous intermediate in the tryptophan metabolic pathway synthesized via the irreversible transamination of its bioprecursor, L-kynurenine by kynurenine aminotransferases [15]. It acts by blocking the co-agonist glycine site of the $\mathrm{N}$-methyl-D-aspartate (NMDA) receptor [16], thereby limiting neurotoxicity resulting from NMDA receptor overstimulation [17]. In addition, KYNA behaves as a noncompetitive inhibitor of the a-7 nicotinic acetylcholine receptor [18] and a ligand for GPR35 localized mainly in gastrointestinal tract and immune cells [19]. These observations indicate the complexity of the neurophysiological actions of KYNA in CNS. In a rat SCI study, local application of KYNA before and early after trauma has been found neuroprotective [20]. The present study has been performed to find out whether posttraumatic application of KYNA, which is more appropriate for clinical use, will have any effect on the outcomes after experimental SCl. We have used a KYNA analogue glucosamine-kynurenic acid, a derivative developed and synthesized in the Institute of Medicinal Chemistry, University of Szeged, Hungary in order to improve the blood-brain barrier transport of KYNA [21,22].

\section{Experimental Procedures}

\subsection{Experimental animals}

A total of 30 male adult Wistar rats weighing from 300 to $330 \mathrm{~g}$ were used in this experiment. All surgical procedures were performed in accordance with the European Communities Council Directive (86/906/EEC) regarding the use of animals in research, and Slovak Law for Animal Protection No. 115/1995.

\subsection{Intrathecal catheter implantation and compression spinal cord injury}

Intrathecal catheter was implanted to all experimental animals 24 hours prior to spinal cord injury. Briefly, under halothane anesthesia (1.5-2\% by facial mask), a PE-5 (4-cm long) sterile polyethylene catheter was introduced through the incision in the atlanto-occipital membrane, threaded down to the Th7-Th8 level of spinal cord, fixed in place by suturing it to the neck superficial muscles and sealed until the time of drug delivery to prevent infection and leakage of cerebrospinal fluid.

Twenty-four hours after the catheter was implanted, animals were evaluated for any motor abnormalities. Only intact animals with normal motor behaviour were included in the study and re-anesthetized. A laminectomy was performed to expose the spinal cord at Th9-10, and SCl was induced by epidural balloon inflation using the method described by Vanicky et al. [23]. Briefly, a 2-French Fogarty catheter (Baxter Healthcare Corporation, Irvine, CA) was inserted via a small hole (1.5 mm diameter) in the Th10 vertebral arch into the epidural space and moved cranially up for $1 \mathrm{~cm}$, with the centre of the balloon at the Th8-9 level. After stabilization of the body temperature at $37^{\circ} \mathrm{C}$, the Fogarty catheter linked to a $50 \mu$ Hamilton syringe (type 1705) was rapidly inflated with $12.5 \mu$ of distilled water for $5 \mathrm{~min}$. Thereafter, the catheter was deflated, removed from epidural space, and the soft tissues and skin were sutured in anatomical layers. The animals were housed individually. Their bladders were expressed manually twice a day for the duration of the study (28 days) or until the recovery of spontaneous micturition, typically in the second week postoperatively.

\subsection{Pharmacological treatment}

The new analogue of KYNA (glucosamine-kynurenic acid) was dissolved in $0.1 \mathrm{M}$ sodium hydroxide, and $\mathrm{pH}$ of the solution was adjusted to 7.4 with $\mathrm{HCl}$ for the final concentration of $50 \mathrm{mM}$. Immediately after injury, spinal catheters were connected via a long tubing to a syringe pump. The tubing was suspended on a balance arm to allow continuous spinal application of the fluid to freely moving animals (Figure 1). Rats were randomly assigned into two groups receiving either glucosaminekynurenic acid $(n=15)$ or saline (control group, $n=15$ ) continually for $24 \mathrm{~h}$ at delivery rate $5 \mu \mathrm{l} / \mathrm{h}$. The total dose od glucosamine-kynurenic acid delivered during $24 \mathrm{~h}$ was $60 \mu \mathrm{mol}$.

\subsection{Evaluation of locomotor recovery}

The locomotion of experimental animals was first assessed 24 hours after injury (day 1 ) and then weekly during the whole survival period. Animals were placed 


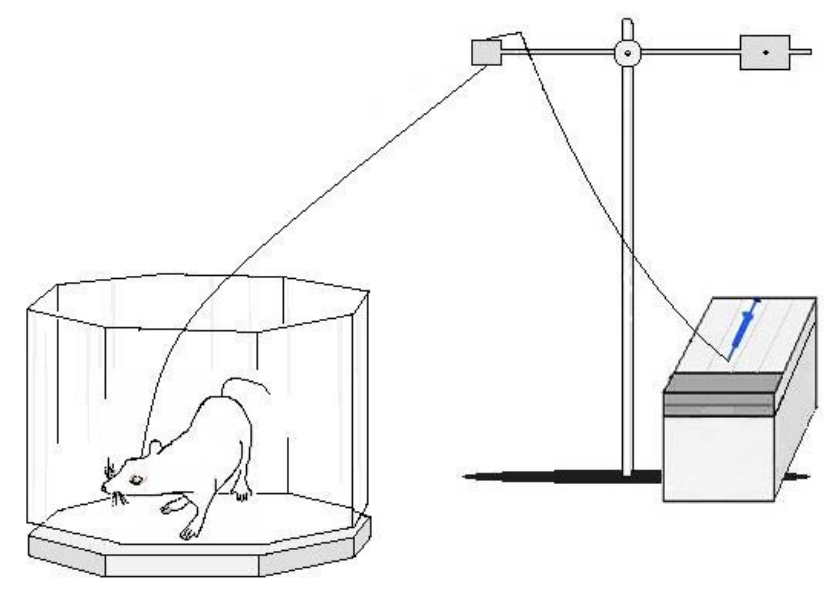

Figure 1. Illustration demonstrating intrathecal drug delivery setup via syringe pump after compression-induced SCI.

in an open field and their spontaneous locomotion was assessed by two observers. Hindlimb movements were evaluated according to the Basso-Beattie-Bresnahan (BBB) scale from 0 to 21 points (from no observable locomotor movements to normal locomotor activity). This scale categorizes combinations of hindlimb joint movements, walking pattern, coordination, trunk stability, paw placement and tail position and stability [24]. As a measure of overall health in both experimental groups, body weights were periodically recorded in the same time intervals as the locomotor recovery tests.

\subsection{Histological and morphometric analysis of spared spinal cord tissue}

After 4 weeks, rats were deeply anesthetized by intraperitoneal injection of ketamine/xylazine $(0.6 \mathrm{ml} / 0.3 \mathrm{ml})$ and perfused transcardially with $0.9 \%$ cold physiological saline followed by $4 \%$ cold paraformaldehyde in $0.1 \mathrm{M}$ phosphate buffer $(\mathrm{pH}$ 7.4). The spinal cords were carefully dissected from the vertebral canal and postfixed by immersion in the same fixative for 24 hours. Dura mater was carefully removed and spinal cords were embedded in a glutaraldehyde-polymerised protein matrix as reported by Herzog and Brosamle [25] in order to facilitate systematic sampling. Consequently, a $3 \mathrm{~cm}$ long piece of the spinal cord (containing the lesion at its center) was cut trasversally with aid of metallic matrix into $1 \mathrm{~mm}$ thick blocks. The blocks marked for proper orientation were flat-embedded in paraffin and $5 \mu \mathrm{m}$ thick sections were cut from the surface of each block. A resulting series of 30 consecutive sections at $1 \mathrm{~mm}$ distances was stained with Luxol Fast Blue/Cresyl Violet. High-resolution images were used to delineate the areas of the preserved tissue. Scar tissue around the cavities and tissues with abnormal architecture were excluded. The crosssectional areas of the white and grey matter were measured using image analysis software (Image Tool for Windows 2.00). From a series of cross-sectional areas, the volume of the spared tissue was calculated. Craniocaudal length of the lesion was measured as a separate morphometric parameter.

\subsection{Statistical analysis}

BBB scores were averaged across hindlimbs to get a single score per animal. Intergroup differences at different times after injury were compared statistically using repeated-measures ANOVA, followed by Tukey's test. Body weight measurements at individual survival time points were compared by the unpaired Student's t-test. For comparison of cross-sectional areas of spared tissue, 27 lesion-centered sections were used. Intergroup differences were evaluated separately for the white matter and grey matter, using repeated-measures ANOVA, followed by Tukey's test. The volume of the preserved tissue in this $27 \mathrm{~mm}$ long segment was calculated as a sum of cross-sectional areas multiplied by the distance between them. Intergroup differences were analyzed with unpaired Student's t-test.

All values were expressed as means \pm S.E.M. Differences between groups were considered statistically significant if $P<0.05$. 


\section{Results}

\subsection{Body weight changes}

The body weight recordings in both experimental groups are shown in Figure 2. After injury, there was a rapid loss in body weight, followed by gradual recovery during the survival period. No significant differences were found between the treatment and placebo groups.

\subsection{Effect of glucosamine-kynurenic acid on Iocomotor activity after SCI}

Immediately after injury, animals in both groups had a complete paralysis of their hindlimbs. During the four weeks' survival period all animals exhibited gradual recovery of their hindlimb movements. By the $7^{\text {th }}$ day post-injury, animals treated with glucosamine-kynurenic acid demonstrated better recovery of motor functions compared to placebo group. The BBB scores were approximately 2 points higher than those in the salinetreated group. These intergroup differences were also observed on days 14, 21 and 28 after injury, and at all these time points the differences reached statistical significance $(P<0.05)$. After 4 weeks post-injury, glucosamine-kynurenic acid treated rats reached BBB score of $14.7 \pm 1.5$, whereas the BBB rating scale for control group was $12.5 \pm 1.3$ (Figure 3 ).

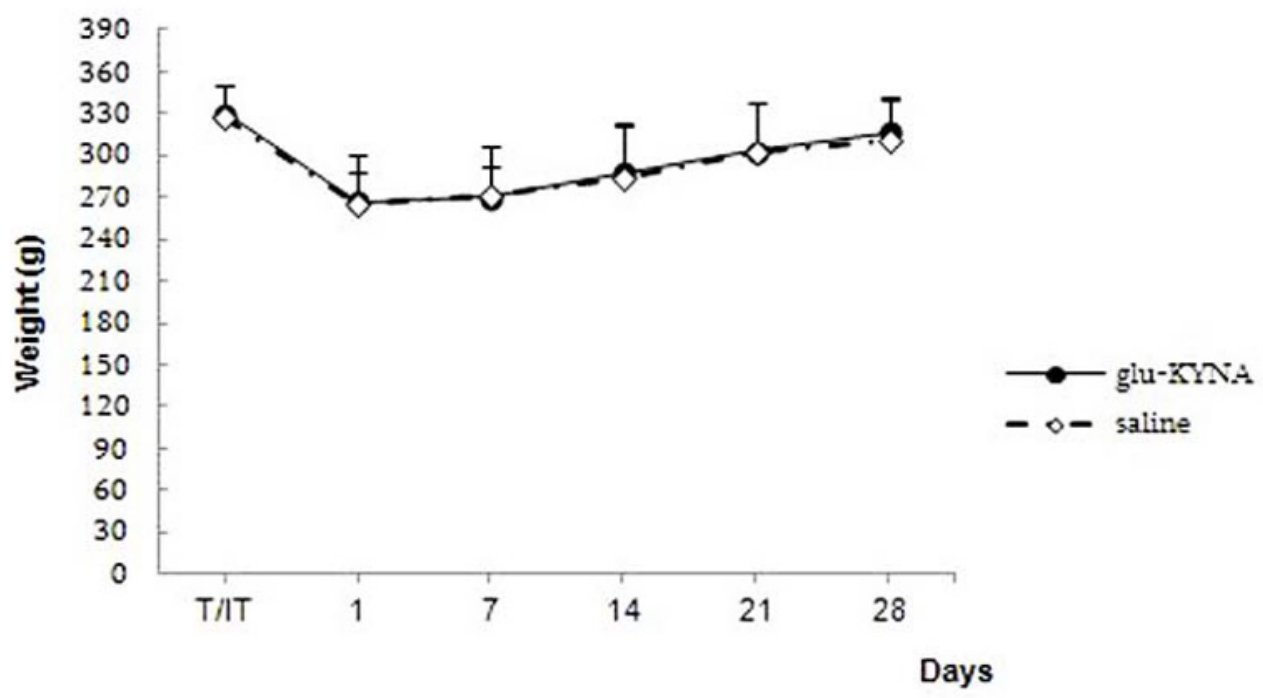

Figure 2. Body weights measured at individual time points (T/IT - trauma/intrathecal drug delivery).

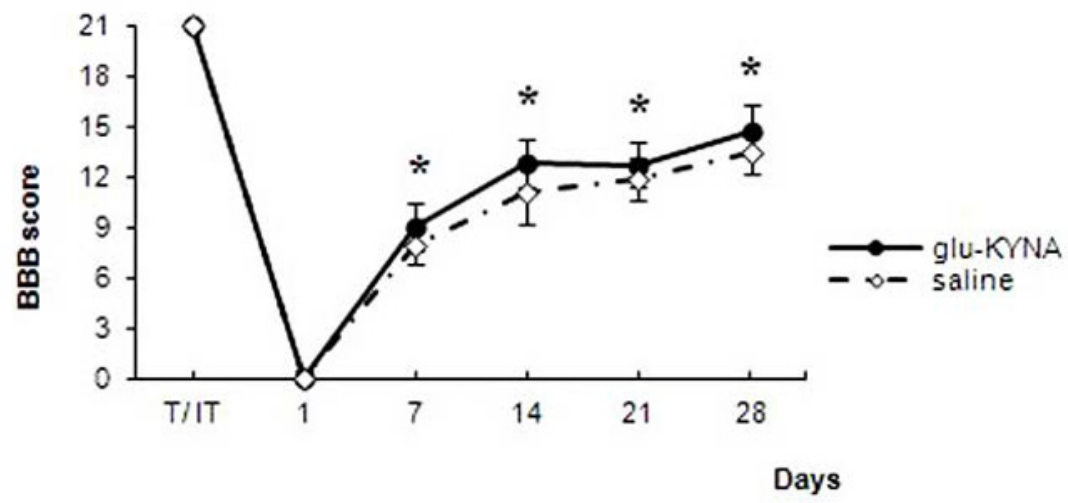

Figure 3. Open field locomotion test. Repeated BBB scores achieved during 28 days' survival period. The rats receiving glucosamine-kynurenic acid exhibited a significant improvement in hindlimb locomotor function compared with the saline control group. Data were analysed with repeated-measures ANOVA which showed an overall significant $(\mathrm{P}<0.05)$ effect of treatment. Asterisks indicate that means are significantly different from control group at the specified times after compression-induced SCI (T/IT - trauma/ intrathecal drug delivery). 


\subsection{Effect of glucosamine-kynurenic acid on the lesion size}

To evaluate the effects of glucosamine-kynurenic acid on the extent of spinal cord tissue damage, complex morphometric analyses of the lesion were performed. A series of cross-sectional areas indicated that generally, there was more tissue preserved in glucosaminekynurenic acid treated group. This tendency was more pronounced in grey matter, and at some levels, these differences reached statistical significance (Figure 4A,B). Total volume calculations also indicated better tissue preservation in the glucosamine-kynurenic acid treated group, but the differences were not statistically significant (Figure 5). The average length of the lesion in control animals was $30.1 \pm 2.1 \mathrm{~mm}$. In glucosamine-kynurenic acid treated animals the lesion length was reduced to $27.7 \pm 1.5 \mathrm{~mm}$, but this difference did not reach statistical significance.

(A)

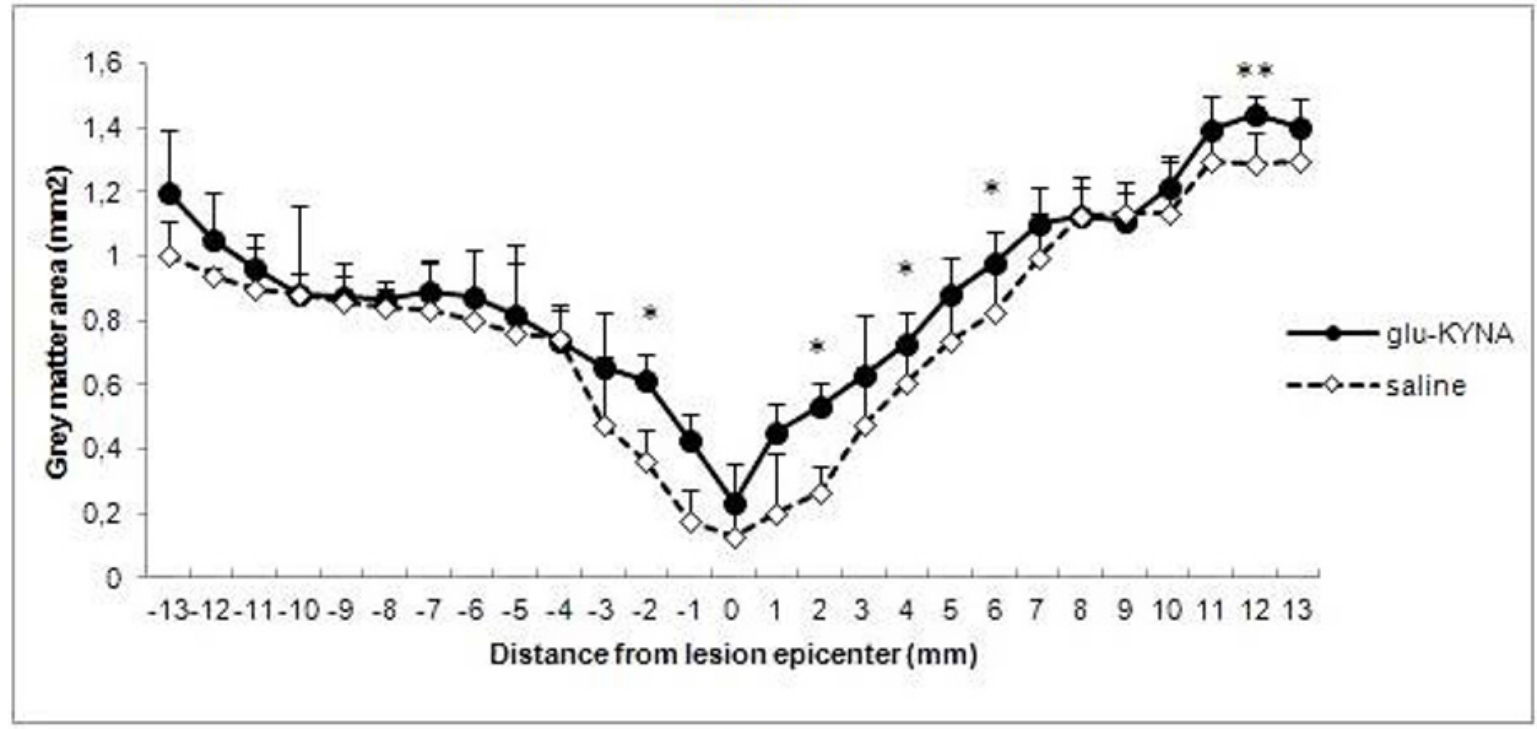

(B)

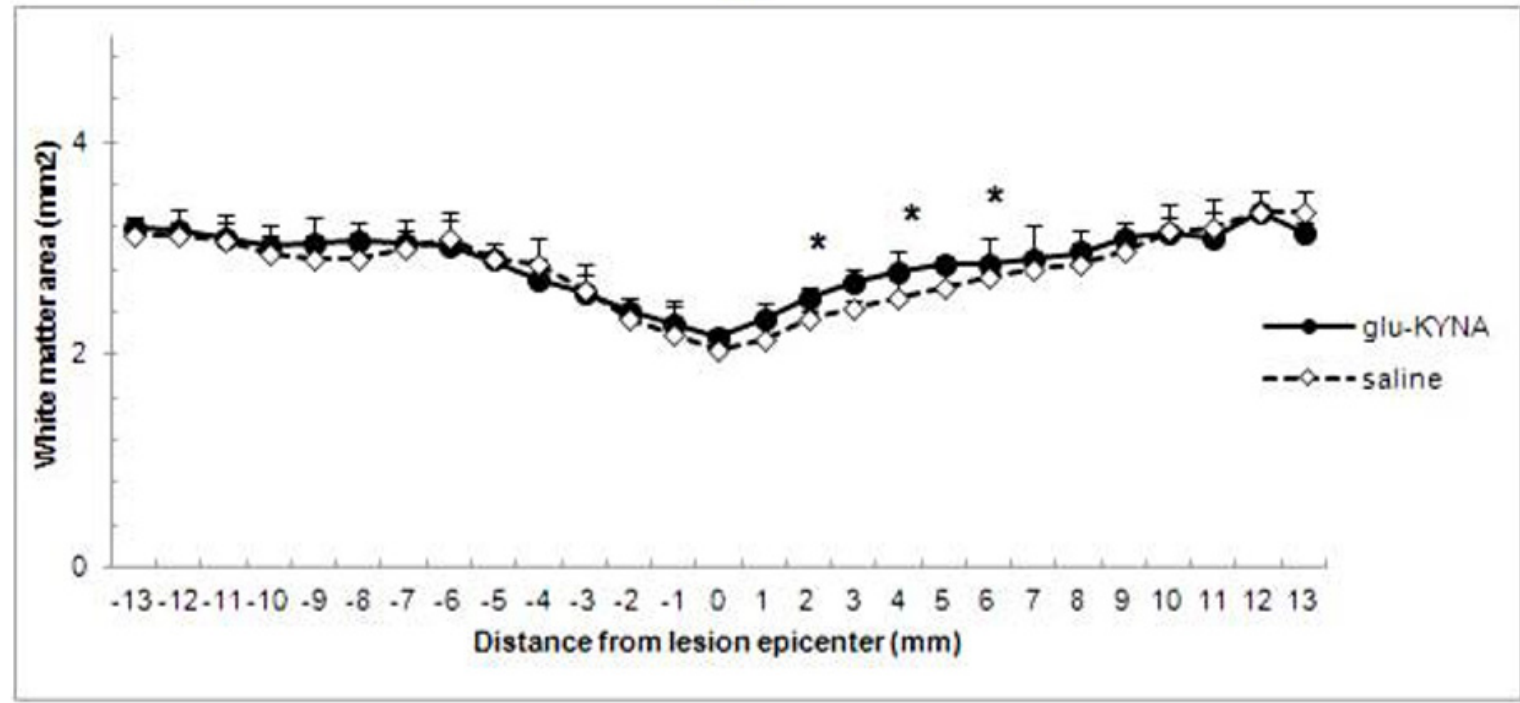

Figure 4. Tissue sparing as assessed by analyzing the cross-sectional areas of spinal cords removed from glucosamine-kynurenic acid and saline treated group after 4 weeks. (A) Grey matter areas in spinal cord sections caudal (-1 to $-13 \mathrm{~mm}$ ) and rostral (1 to $13 \mathrm{~mm})$ to the lesion epicenter. (B) White matter areas at the epicenter and in sections caudal and rostral to the epicenter. Repeated-measures ANOVA indicated an overall significant differences in grey and white matter area between the glucosamine-kynurenic acid treated and the control group, with significant differences at specified distances to the epicenter ( ${ }^{\star} P<0.05,{ }^{\star}{ }^{\star} \mathrm{P}<0.01$ ). 


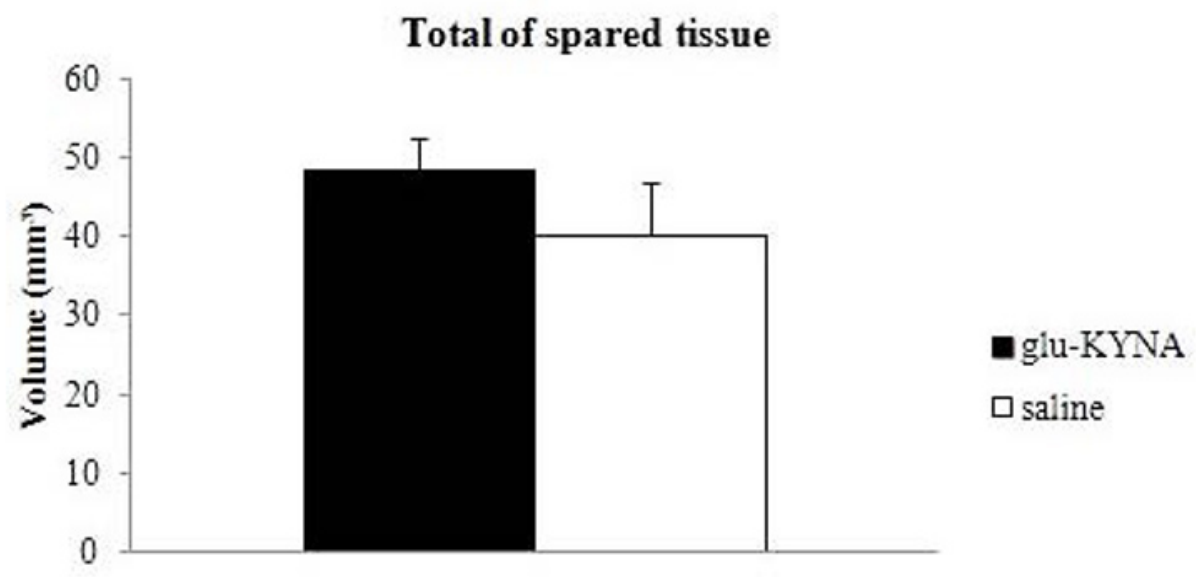

Total

Figure 5. Calculated volumes of the spared tissue in the analysed spinal cord segments $(27 \mathrm{~mm})$ in glucosamine-kynurenic acid and saline treated group. No significant treatment effect was seen. Data are represented as group means \pm SEM.

\section{Discussion}

As early as $15 \mathrm{~min}$ after $\mathrm{SCl}$, extracellular $\mathrm{EAA}$ concentrations can rise to the levels that are apparently toxic to neurons $[26,27]$. It has been proposed that the increase in extracellular EAAs after traumatic $\mathrm{SCl}$ allows excessive $\mathrm{Ca}^{2+}$ and water influx into the neurons mainly via the NMDA receptors, resulting in cellular edema and neuronal death [28]. Thus, overactivation of EAA receptors is considered to be one of the major contributors to the pathophysiological cascade leading to tissue destruction after traumatic SCI [29], and EAA receptor antagonists have been the most potent neuroprotectants in experimental SCI treatment studies.

In the present study we have tested the neuroprotective effects of a glucosamine-kynurenic acid on the functional outcome and spinal lesion size after standardized $\mathrm{SCl}$ in the rat. KYNA is the only known endogeneous NMDA receptor inhibitor and thus represents a potential therapeutic agent in various neurodegenerative diseases where excitotoxicity is considered to play a role in pathophysiological cascade. However, KYNA has a limited ability to cross the bloodbrain barrier. A new KYNA analogue, glucosaminekynurenic acid has been synthesized in the Institute of Medicinal Chemistry, University of Szeged, Hungary, with the aim to prepare a drug that would cross the blood-brain barrier better than KYNA, while maintaining its effects on CNS when applied either peripherally or directly into the CNS [21,30].

The aim of this treatment study was twofold: 1 , to test the neuroprotective effects of the new KYNA analogue after CNS trauma, and 2, to determine whether a clinically relevant administration (continual spinal infusion during 24 hours after injury) can influence the long-term consequences of $\mathrm{SCl}$. We have used the balloon-compression technique of $\mathrm{SCl}$, representing a highly reliable and reproducible $\mathrm{SCl}$ model [23], associated with the interruption of the major long spinal tracts (dorsal funiculi, pyramidal tracts) at the injury epicenter.

The results of our experiments have shown that intrathecal infusion of glucosamine-kynurenic acid is a safe technique of long-term drug application. In glucosamine-kynurenic acid treated animals we have observed a modest but significant improvement in locomotor recovery ( 2 points in BBB score) detected first after 7 days and then persisting throughout the whole survival period. Morphometric data indicated overall improvement in perilesional tissue preservation in the glucosamine-kynurenic acid treated group. The improved tissue preservation was more pronounced in the grey matter, but also in the white matter, there was more preserved tissue almost at all levels throughout the lesion. At some points these differences reached statistical significance. In addition, both total volume of the spared tissue calculations and craniocaudal length of the lesion indicated improved outcomes in glucosamine-kynurenic acid treated animals although these differences did not reach statistical significance.

Our findings are consistent with earlier observations that peri-traumatic EAA receptor blockade after experimental $\mathrm{SCl}$ can improve functional deficits caused by injury $[20,31,32]$. It has been demonstrated that experimental CNS traumatic injury is associated with several-fold increase in extracellular EAAs, especially glutamate and aspartate [12,26,33]. This increase in extracellular concentrations is caused by 
a combination of impaired glutamate reuptake and calcium-dependent glutamate synaptic release. High levels of extracellular glutamate concentration are toxic to neural cells. Especially in hypoxic conditions, ionotropic glutamate receptors, i.e. NMDA and AMPA/kainate are overstimulated and can trigger the process of excitotoxic neuronal death [34,35]. Mechanisms of excitotoxicity are complex, but are believed to involve excessive intracellular $\mathrm{Ca}^{2+}$ influx associated with mitochondrial injury and impaired energy production, triggering a vicious cycle leading to cellular edema and lysis $[36,37]$. This cascade is relatively well understood in grey matter. However, after $\mathrm{SCl}$, mechanisms leading to secondary white matter destruction are even more important. It has been long believed that white matter is resistant to excitotoxic injury, as it does not contain synapses. However, in vitro experiments indicate that under anoxic conditions, glutamate levels increase and may destroy the white matter structures as well. Prolonged exposition of central white matter to increased concentrations of glutamate, AMPA and kainate brings about irreversible axonal injury mediated primarily via non-NMDA receptors [38,39]. These findings were supported by in vivo observations documenting that antagonists of AMPA receptors reduce the lesion size in the epicenter, and improve neurological

\section{References}

[1] Crowe M.J., Bresnahan J.C., Shuman S.L., Masters J.N., Beattie M.S., Apoptosis and delayed degeneration after spinal cord injury in rats and monkeys, Nat. Med., 1997, 3, 73-76

[2] Tator C.H., Fehlings M.G., Review of the secondary injury theory of acute spinal cord trauma with emphasis on vascular mechanisms, J. Neurosurg., 1991,75, 15-26

[3] Fitch M.T., Doller C., Combs C.K., Landreth G.E., Silver J., Cellular and molecular mechanisms of glial scarring and progressive cavitation: in vivo and in vitro analysis of inflammation-induced secondary injury after CNS trauma, J. Neurosci., 1999, 19, 8182-8198

[4] Nelson E., Gertz S.D., Rennels M.L., Ducker T.B., Blaumanis O.R., Spinal cord injury. The role of vascular damage in the pathogenesis of central hemorrhagic necrosis, Arch. Neurol., 1977, 34, 332-333

[5] Taoka Y., Okajima K., Spinal cord injury in the rat, Prog. Neurobiol., 1998, 56, 341-358

[6] Liu X.Z., Xu X.M., Hu R., Du C., Zhang S.X., McDonald J.W., et al., Neuronal and glial apoptosis outcomes after SCI [32]. Rosenberg et al. [40] have demonstrated that AMPA blockers can reduce the amount of injured white matter and oligodendrocyte loss after spinal cord traumatic injury.

In general, the results of our study support the importance of EAA receptor blockade in functional recovery after spinal cord injury. We have shown that continuous local delivery of glucosamine-kynurenic acid via intrathecal catheter after experimental spinal cord injury is a safe treatment which can lead to behavioral improvements and prevent secondary destruction of the spinal tissue. It supports the view that secondary processes after spinal trauma mediated via excitotoxic mechanisms play an important role in tissue destruction. Secondary extension of the lesion is a relatively slow process that contributes to the final functional deficit, and provide a therapeutic window for specific treatment interventions.

\section{Acknowledgements}

This work was supported by the following grant: VEGA $2 / 0202 / 10$. We gratefully acknowledge the assistance of Viera Kujaníková in preparing and staining histological sections. We thank Csaba Somai for synthesizing the glucosamine-kynurenic acid.

after traumatic spinal cord injury, J. Neurosci., 1997, 17, 5395-5406

[7] Tator C.H., Koyanagi I., Vascular mechanisms in the pathophysiology of human spinal cord injury, J. Neurosurg., 1997, 86, 483-492

[8] Balentine J.D., Pathology of experimental spinal cord trauma. I. The necrotic lesion as a function of vascular injury, Lab. Invest., 1978, 39, 236-253

[9] Kwon B.K., Okon E., Hillyer J., Mann C., Baptiste D., Weaver L.C., et al., A systematic review of noninvasive pharmacologic neuroprotective treatments for acute spinal cord injury, J. Neurotrauma, 2011, 28, 1545-1588

[10] Bracken M.B., Shepard M.J., Collins W.F., Holford T.R., Young W., Baskin D.S., et al., A randomized, controlled trial of methylprednisolone or naloxone in the treatment of acute spinal-cord injury. Results of the Second National Acute Spinal Cord Injury Study, N. Engl. J. Med., 1990, 322, 1405-1411

[11] Short D.J., El Masry W.S., Jones P.W., High dose methylprednisolone in the management of acute 
spinal cord injury - a systematic review from a clinical perspective, Spinal Cord, 2000, 38, 273-286

[12] McAdoo D.J., Xu G.Y., Robak G., Hughes M.G., Changes in amino acid concentrations over time and space around an impact injury and their diffusion through the rat spinal cord, Exp. Neurol., 1999, 159, 538-544

[13] Ikonomidou C., Turski L., Excitotoxicity and neurodegenerative diseases, Curr. Opin. Neurol., 1995, 8, 487-497

[14] Muir K.W., Lees K.R., Clinical experience with excitatory amino acid antagonist drugs, Stroke, 1995, 26, 503-513

[15] Stone T.W., Kynurenines in the CNS: from endogenous obscurity to therapeutic importance, Prog. Neurobiol., 2001, 64, 185-218

[16] Birch P.J., Grossman C.J., Hayes A.G., Kynurenic acid antagonises responses to NMDA via an action at the strychnine-insensitive glycine receptor, Eur. J. Pharmacol., 1988, 154, 85-87

[17] Schwarcz R., Pellicciari R., Manipulation of brain kynurenines: glial targets, neuronal effects, and clinical opportunities, J. Pharmacol. Exp. Ther., 2002, 303, 1-10

[18] Hilmas C., Pereira E.F., Alkondon M., Rassoulpour A., Schwarcz R., Albuquerque E.X., The brain metabolite kynurenic acid inhibits alpha7 nicotinic receptor activity and increases non-alpha7 nicotinic receptor expression: physiopathological implications, J. Neurosci., 2001, 21, 7463-7473

[19] Wang J., Zhao X., Zheng Y., Kong H., Lu G., Cai Z., et al., Kynurenic acid as a ligand for orphan $G$ protein-coupled receptor GPR35, J. Biol. Chem., 2006, 281, 22021-22028

[20] Wrathall J.R., Bouzoukis J., Choiniere D., Effect of kynurenate on functional deficits resulting from traumatic spinal cord injury, Eur. J. Pharmacol., 1992, 218, 273-281

[21] Fuvesi J., Somlai C., Nemeth H., Varga H., Kis Z., Farkas T., et al., Comparative study on the effects of kynurenic acid and glucosamine-kynurenic acid, Pharmacol. Biochem. Behav., 2004, 77, 95-102

[22] Nemeth H., Toldi J., Vecsei L., Role of kynurenines in the central and peripheral nervous systems, Curr. Neurovasc. Res., 2005, 2, 249-260

[23] Vanicky I., Urdzikova L., Saganova K., Cizkova D., Galik J., A simple and reproducible model of spinal cord injury induced by epidural balloon inflation in the rat, J. Neurotrauma, 2001, 18, 1399-13407

[24] Basso D.M., Beattie M.S., Bresnahan J.C., A sensitive and reliable locomotor rating scale for open field testing in rats, J. Neurotrauma, 1995, 12, $1-21$
[25] Herzog A., Brosamle C., 'Semifree-floating' treatment: a simple and fast method to process consecutive sections for immunohistochemistry and neuronal tracing, J. Neurosci. Methods, 1997, 72, 57-63

[26] Liu D., Thangnipon W., McAdoo D.J., Excitatory amino acids rise to toxic levels upon impact injury to the rat spinal cord, Brain Res., 1991, 547, 344348

[27] Painter S.C., Wum S.W., Faden A.I., Alteration in extracellular amino acids after traumatic spinal cord injury, Ann. Neurol., 1990, 27, 96-99

[28] Mody I., MacDonald J.F., NMDA receptordependent excitotoxicity: the role of intracellular Ca2+ release, Trends Pharmacol. Sci., 1995, 16, 356-359

[29] Hall E.D., Springer J.E., Neuroprotection and acute spinal cord injury: a reappraisal, NeuroRx., 2004, 1, 80-100

[30] Robotka H., Németh H., Somlai C., Vécsei L., Toldi J., Systemically administered glucosaminekynurenic acid, but not pure kynurenic acid, is effective in decreasing the evoked activity in area CA1 of the rat hippocampus, Eur. J. Pharmacol., 2005, 513, 75-80

[31] Faden A.I., Ellison J.A., Noble L.J., Effects of competitive and non-competitive NMDA receptor antagonists in spinal cord injury, Eur. J. Pharmacol., 1990, 175, 165-174

[32] Wrathall J.R., Choiniere D., Teng Y.D., Dosedependent reduction of tissue loss and functional impairment after spinal cord trauma with the AMPA/ kainate antagonist NBQX, J. Neurosci., 1994, 14, 6598-6607

[33] McAdoo D.J., Wu P., Microdialysis in central nervous system disorders and their treatment, Pharmacol. Biochem. Behav., 2008, 90, 282-296

[34] Carriedo S.G., Yin H. Z., Sensi S. L., Weiss J. H. (1998) Rapid Ca2+ entry through Ca2+-permeable AMPA/Kainate channels triggers marked intracellular $\mathrm{Ca} 2+$ rises and consequent oxygen radical production, J. Neurosci., 1998, 18, 77277738

[35] Doble A., The role of excitotoxicity in neurodegenerative disease: implications for therapy, Pharmacol. Ther., 1999, 81, 163-221

[36] Choi W.D., Excitotoxic cell death, J. Neurobiol., 1992, 23, 1261-1276

[37] Choi W.D., Glutamate neurotoxicity and diseases of the nervous system, Neuron, 1998, 1, 623-634

[38] Li S., Mealing G.A., Morley P., Stys P.K., Novel injury mechanism in anoxia and trauma of spinal cord white matter: glutamate release via reverse 
$\mathrm{Na}+$-dependent glutamate transport, J. Neurosci., 1999, 19, RC16

[39] Li S., Stys P.K., Mechanisms of ionotropic glutamate receptor-mediated excitotoxicity in isolated spinal cord white matter, J. Neurosci., 2000, 20, 1190-1198
[40] Rosenberg L.J., Teng Y.D., Wrathall J.R., 2,3-Dihydroxy-6-nitro-7-sulfamoyl-benzo(f) quinoxaline reduces glial loss and acute white matter pathology after experimental spinal cord contusion, J. Neurosci., 1999, 19, 464-475 